INRA Prod. Anim., 1998, 11 (5), 349-357

\author{
V. NOIROT(1), I. BOUVAREL ${ }^{(1)}$, \\ B. BARRIER-GUILLOT ${ }^{(2)}$, \\ J. CASTAING ${ }^{(3)}$, J.L. ZWICK ${ }^{(4)}, M$. \\ PICARD ${ }^{(5)}$ \\ (1) ITAVI 28 rue du Rocher 75008 Paris, \\ (2) ITCF 91720 Boigneville, \\ (3) AGPM Route de Pau 64121 Montardon, (4) UCAAB \\ 02400 Chateau-Thierry, \\ (5) INRA-SRA 37380 Nouzilly
}

\section{Céréales entières pour les poulets de chair : le retour?}

Les céréales représentent 60 à $70 \%$ des 3,4 millions de tonnes d'aliment composé fabriquées en France en 1997 pour les poulets de chair. Il y a 50 ans, ces granivores consommaient des graines de céréales entières dans les basses-cours. Actuellement, les céréales sont broyées, mélangées avec d'autres matières premières, pour obtenir un aliment complet unique qui est ensuite granulé. Dans les grands élevages d'Europe du nord, des céréales entières (blé principalement), sont distribuées simultanément ou alternativement avec un aliment complémentaire. Ce mode d'alimentation permet d'adapter la proportion de céréales consommées à l'âge du poulet et de diminuer les coûts de stockage, de transformation et de transport des céréales si l'aviculteur les produit.

Les résultats de la recherche permettent de comparer les trois principales méthodes d'utilisation des graines entières dans l'alimentation du poulet de chair : distributions simultanée mais séparée dans l'espace, en mélange, ou séquentielle avec un aliment complémentaire.

\section{Résumé}

L'utilisation des céréales entières distribuées avec un aliment complémentaire dans l'alimentation du poulet de chair présente un regain d'intérêt avec les succès rencontrés dans certains élevages d'Europe du nord. Les résultats de la recherche permettent de comparer les trois principaux modes de distribution des deux aliments : l'alimentation séparée dans l'espace, le mélange et l'alimentation séquentielle.

Mélange et alimentation séquentielle sont adaptés à l'élevage : ces techniques permettent de mieux contrôler les proportions de céréale et d'aliment complémentaire consommées par les poulets. L'alimentation séparée laisse en revanche s'exprimer les facteurs individuels de la sélection alimentaire et ne permet pas toujours d'obtenir la croissance et la composition corporelle escomptées par l'éleveur.

Le poulet digère aussi bien les céréales entières que broyées. Il est capable de s'adapter rapidement à un régime comportant une céréale entière, en modifiant son comportement alimentaire. La présentation physique et la composition de l'aliment complémentaire affectent la proportion de céréales consommée. Les céréales entières induisent un développement du gésier, dont les effets sur la digestion et la prévention de la coccidiose méritent d'être approfondis.

La technique du mélange est maîtrisée et appliquée dans les élevages d'Europe du nord, avec des systèmes de distribution informatisés. Il reste à valider en pratique le mode de distribution séquentiel, moins coûteux que le mélange, et à étudier l'intérêt économique réel de cette méthode d'alimentation pour l'éleveur de poulet en France.
Le développement de l'utilisation des céréales entières en élevage dépend de la maîtrise technique des méthodes de distribution. Les trois principales sont l'alimentation séparée, la distribution séquentielle et le mélange (figure 1). Les techniques de distribution de céréales graines entières sont donc toutes basées sur un choix plus ou moins dirigé de l'animal dans l'espace, le temps ou par tri particulaire.

Un poulet au sein d'un groupe est capable de sélectionner un régime globalement équilibré, lorsqu'il est confronté à différents aliments d'égale disponibilité dans son environnement (Covasa et Forbes 1995). Le choix initial est celui de consommer ou non une particule présente dans cet environnement. L'identification de l'aliment est une capacité acquise par l'apprentissage (Picard 1997). Il permet à l'animal d'associer les critères de différenciations sensorielles (forme, couleur, odeur, saveur, propriétés tactiles) et les caractéristiques nutritionnelles des aliments " goûtés ». L'équilibrage du niveau de consommation de deux aliments ne se fait que si ces derniers diffèrent par au moins une caracté- 
Figure 1. Les trois principales méthodes de distribution de céréales graines entières.

\begin{tabular}{|lll|}
\hline $\begin{array}{l}\text { Alimentation séparée dans l'espace } \\
\text { types d'aliments présentés simultanément } \\
\text { dans des mangeoires distinctes. }\end{array}$ & $\begin{array}{c}\text { Méréale } \\
\square \text { Climent } \\
\text { complémentaire }\end{array}$ & $\begin{array}{l}\text { Présentation simultanée des deux aliments } \\
\text { dans la même mangeoire permettant de } \\
\text { contrôler en partie la sélection alimentaire avec } \\
\text { des proportions de céréales déterminées et } \\
\text { variables avec l'âge du poulet. Le poulet reste } \\
\text { libre de sélectionner les particules dans le } \\
\text { mélange. }\end{array}$ \\
\end{tabular}

ristique nutritionnelle (Forbes et Kyriasakis 1995) et s'il existe au moins une solution équilibrée dans le choix offert (Emmans 1991). L'offre, simultanée ou non, de blé riche en énergie et pauvre en protéines et d'un concentré hautement protéique, riche en minéraux, oligo-éléments et vitamines, et moins riche en énergie, remplit ces conditions.

Les choix alimentaires dépendent de trois grands types de facteurs schématisés à la figure 2 (Covasa et Forbes 1995, MeunierSalaün et Picard 1996, Yo 1996). L'interprétation des choix alimentaires des animaux est souvent confuse. Le « bon » choix pour le poulet n'est pas forcément celui qui lui permet de maximiser sa croissance (Siegel et al 1997). Le principe du choix offre cependant l'avantage d'une flexibilité dans la composition du régime, répondant à la variabilité des conditions d'ambiance. Dans le cas particulier de l'offre de graines entières et d'un concentré protéique, les poulets ont la possibilité de réguler leur ingéré protéique indépendamment de l'ingestion d'énergie et de l'adapter à leur niveau de production ainsi qu'aux variations nycthémérales de température (Mastika et Cumming 1987). Cependant, plusieurs questions font encore l'objet d'un débat scientifique : à quel âge, à quel niveau d'incorporation dans le régime global, et comment introduire les céréales entières pour obtenir des performances comparables à celles observées avec un aliment complet? Quelles sont ensuite les conséquences de ce type d'alimentation sur le comportement alimentaire et la santé du poulet?

Les principaux avantages et inconvénients des trois principales techniques sont résumés, en considérant successivement l'alimentation séparée, le mélange, illustré par des exemples de techniques utilisées en élevage en Europe du nord, et l'alimentation séquentielle. Quelques résultats récents de la recherche sont ensuite analysés pour faire le point sur les développements possibles de la distribution des céréales entières aux poulets de chair.

\section{1 / Avantages et inconvénients des trois méthodes d'alimentation}

Le but recherché est d'obtenir des vitesses de croissance, efficacité alimentaire et composition corporelle les plus proches possibles de celles de poulets consommant un aliment complet, en utilisant des céréales entières et un aliment complémentaire riche en protéines.

\section{1 / Lalimentation séparée dans l'espace}

Les résultats de l'alimentation séparée sont très variables, en termes de performances de croissance, de qualité des carcasses et de niveau de consommation de la céréale entière. Dans des conditions expérimentales diverses (climat, logement, type de céréale, composition de l'aliment complémentaire, âge à l'introduction de la céréale), des résultats globalement encourageants sont parfois obtenus : par exemple, avec du blé entier entre 18 et 46 jours et un programme lumineux alterné avec une heure d'éclairage toutes les quatre heures (Rose et Lambie 1986), du sorgho entier à partir de 10 jours (Mastika et Cumming 1987), du blé entier entre 7 et 49 jours (Leeson et Caston 1993) ou, enfin, du maïs, en farine jusqu'à 21 jours, puis entier jusqu'à 56 jours en climat chaud (Yo et al 1994). Dans ces exemples, les vitesses de croissance sont semblables à celles obtenues avec un aliment complet. Mais d'autres auteurs rapportent des échecs de l'alimentation séparée : par exemple 
Figure 2. Facteurs modifiant les choix alimentaires du poulet de chair (d'après Yo 1996).

Le poulet sélectionne les aliments en établissant le lien entre leurs propriétés nutritionnelles et leurs caractéristiques sensorielles, perçues par la vision, l'olfaction et le toucher. La sélection dépend en partie des besoins de l'animal, qui varient selon le sexe, la souche et le stade physiologique. La capacité de choix est renforcée par une période d'apprentissage. Le milieu de vie aussi est déterminant : la température ambiante ainsi que la situation physique des aliments offerts (mangeoires différentes ou non, position relative des mangeoires et espace relatif occupé par les différentes fractions alimentaires) modulent les choix. La vie en groupe permet une meilleure acquisition de l'aptitude à une sélection alimentaire adaptée, par imitation des congénères. La sélection alimentaire est en définitive le résultat d'un compromis entre l'appétence relative des aliments proposés et les besoins du poulet dans un environnement donné.

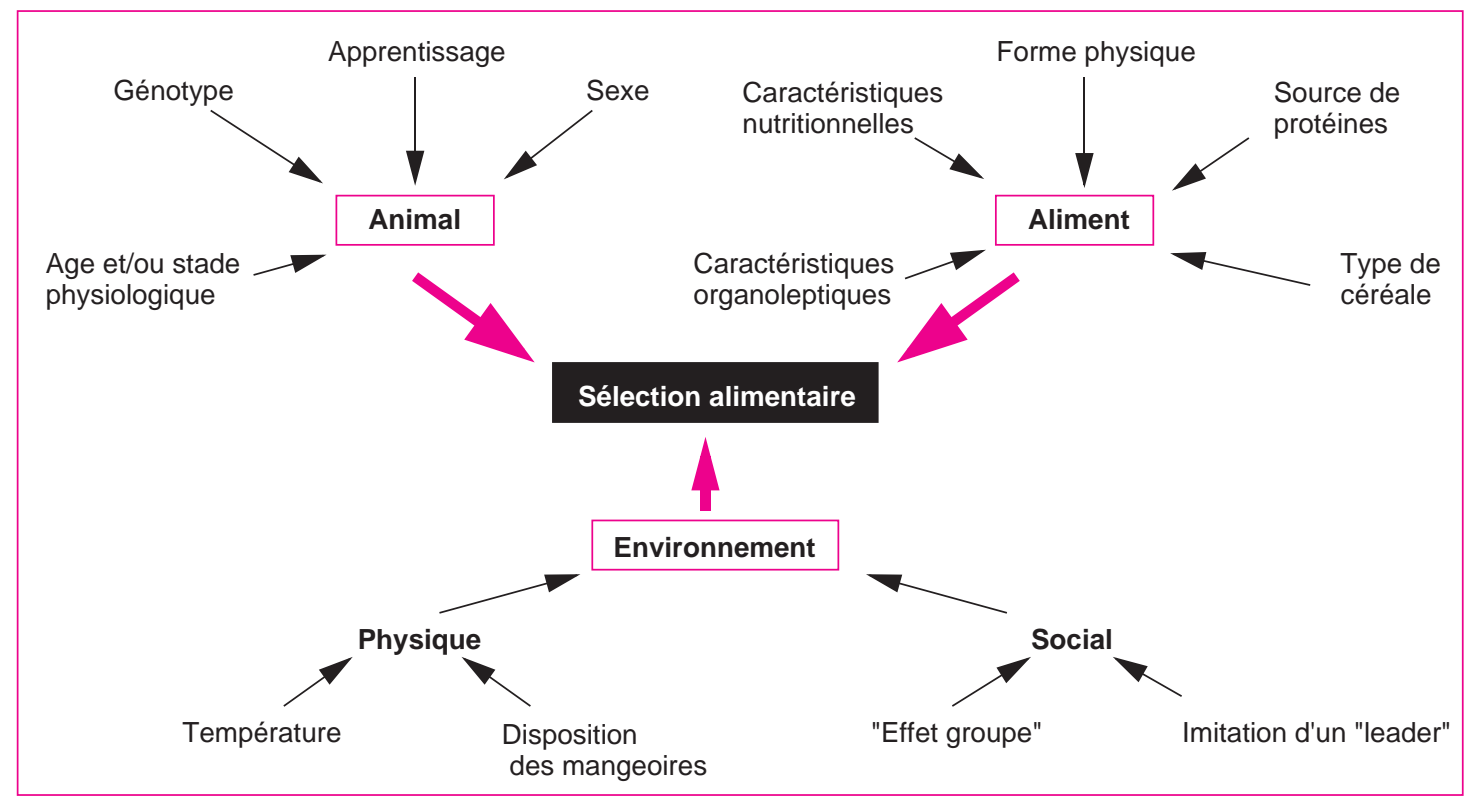

les poids vifs à 42 jours sont inférieurs de 5 à $7 \%$ à ceux des poulets recevant un aliment complet, lorsque les poulets reçoivent du blé entier entre 14 et 42 jours et un aliment complémentaire titrant $30 \%$ de protéines brutes (Scholtyssek et al 1983), ou un mélange de sorgho et de blé entiers entre 21 et 42 jours, avec un aliment complémentaire à $40 \%$ de protéines brutes (Munt et al 1995).

Comment expliquer la variabilité des résultats ? La description de quelques situations nutritionnelles étudiées en alimentation séparée permet de discuter de l'influence de deux types de facteurs de variation.

\section{a / Teneur en protéines de l'aliment complémentaire et équilibre du régime}

Globalement, il semble que plus l'aliment complémentaire est riche en azote et pauvre en énergie par rapport à la céréale, plus la proportion de céréale entière consommée est élevée. Ainsi, avec des teneurs de 40 à $50 \%$ de protéines brutes dans l'aliment complémentaire, des niveaux de consommation de céréale entière de l'ordre de 60-70\% du régime global sont obtenus avec du blé entier (Cowan et Mitchie 1978), du sorgho entier (Mastika et Cumming 1987), ou du maïs sous forme de farine puis entier (Yo et al 1994). En revanche, lorsque la teneur en protéines brutes de l'aliment complémentaire est plus faible (22\%), et sa teneur en énergie métabolisable proche de celle de la céréale (3050 $\mathrm{kcal} / \mathrm{kg}$ ), la consommation de blé entier ou de maïs concassé atteint seulement $30 \%$ en moyenne chez des poulets de 7 à 49 jours d'âge (Leeson et Caston 1993).

Les céréales se différenciant des aliments complémentaires par leurs teneurs en protéines et en énergie métabolisable, le principal problème rencontré est celui du rapport protéines/énergie du régime effectivement ingéré par le poulet. Il peut être différent de celui d'un aliment complet et ne pas permettre d'obtenir les mêmes performances de croissance et /ou des qualités de carcasse comparables. Par exemple, dans le cas où une part importante de maïs concassé est consommée entre 1 et 42 jours (73\%, avec un concentré à $44 \%$ de protéines brutes), une consommation protéique inférieure de $15 \%$ à celle du témoin recevant un aliment complet est constatée, les consommations d'énergie étant comparables. L'efficacité alimentaire, protéique ou énergétique n'est dans ce cas pas affectée par la technique du choix alimentaire, mais la vitesse de croissance des poulets est réduite de $10 \%$ (Yo et al 1997). Dans d'autres cas, les performances de croissance ne sont pas différentes de celles du témoin, mais une surconsommation énergétique et une sous-consommation protéique entraînent une augmentation de la teneur en gras abdominal de la carcasse et une diminution des

\section{En alimentation séparée, la consommation de céréales varie selon les teneurs en protéines et en énergie de l'aliment complémentaire.}


rendements en filets (Leeson et Caston 1993). Un déficit de $20 \mathrm{~g}$ de protéines pour 2390 kcal d'énergie métabolisable est responsable d'une augmentation d'un point de gras abdominal par rapport au poids de carcasse (Scholtyssek et al 1983).

Une question importante reste à élucider dans ces études : l'origine des variations de choix des poulets est-elle protéique ou énergétique ? Il y a en effet concomitance entre la réduction de la concentration énergétique et l'augmentation de la concentration protéique de l'aliment complémentaire.

\section{b / Localisation des aliments dans l'espace et mémoire}

La sélection se faisant dans l'espace, une préférence artificielle pour l'un des deux types d'aliments pourrait provenir de leurs localisations respectives dans le lieu de vie. Un rapport «espace occupé par la céréale entière : espace occupé par le concentré » variable $(2: 1$, $1: 1,1: 2)$ ne semble pas avoir beaucoup d'influence sur la proportion de chacun des deux aliments sélectionnée par des poulets de chair entre 21 et 49 jours d'âge (Rose et al 1986). Il semble cependant que la localisation des aliments soit un élément d'identification pour le poulet. Si on inverse la position des mangeoires après 3 semaines d'adaptation à un régime basé sur le choix alimentaire, la corrélation entre la quantité ingérée dans l'ancienne position la semaine précédant le changement et celle consommée dans la nouvelle position est de 0,9 le septième jour après l'inversion des positions (Rose et Kyriasakis 1991).

Le principal avantage de la méthode d'alimentation séparée est la simplicité de sa mise en œuvre pratique. L'espace relatif à occuper par chacun des deux aliments doit être cependant déterminé en conditions d'élevage. Il reste que la sélection des deux types d'aliment est difficilement maîtrisable, d'où parfois une détérioration des résultats.

\section{2 / Le mélange céréale entière + aliment complémentaire}

\section{a / Résultats de la recherche}

Les auteurs travaillant sur cette technique utilisent, avec du blé entier, soit un aliment complet traditionnel (Leeson et Caston 1993, Covasa et Forbes 1994), soit un aliment complémentaire plus riche en protéines (Rose et al 1995). Cette technique permet de maîtriser en moyenne les niveaux d'ingestion des différents nutriments : le contenu protéique des aliments offerts en mélange à des poulets de chair entre 0 et 49 jours ( 2 à $34 \%$ de blé incorporés dans un aliment complet) correspond bien à la quantité de protéines consommée au cours de la période (Covasa et Forbes 1994). Les performances de croissance sont comparables à celles obtenues avec un aliment complet unique. Des poulets recevant un mélange de blé entier et d'un aliment complémentaire titrant $30 \%$ de protéines brutes et $3060 \mathrm{kcal} / \mathrm{kg}$ entre 24 et 45 jours d'âge, avec un taux d'incorporation de blé croissant de 40 à $60 \%$, ont les mêmes gain de poids et indice de consommation que ceux recevant un aliment complet au cours de la même période (Rose et al 1995).

\section{b / Applications en élevage}

Le mélange est la technique la plus couramment pratiquée dans les élevages utilisant les graines entières de blé, en Europe du nord. En 1997, environ 400 élevages européens utilisent avec succès cette technique, avec un système de distribution informatisé, principalement en Grande-Bretagne où la proportion d'élevages équipés est de $10 \%$ (Filmer 1996, Le Douarin 1997). En 1995, 5 \% des aviculteurs des Pays-Bas utilisaient du blé entier (Montjoie 1995). En France, cette technique est encore peu développée, elle est simplement testée dans quelques élevages.

L'aliment complémentaire peut être un aliment complet de démarrage, dilué progressivement avec du blé, ce qui permet une incorporation moyenne de $15 \%$ de céréales entières pour des poulets de $2 \mathrm{~kg}$ à l'abattage (Le Douarin 1997). Si un aliment complémentaire riche en protéines est utilisé, le blé peut être incorporé jusqu'à $30 \%$ en moyenne (Montjoie 1995, Gérard 1997).

Des systèmes de régulation informatique des proportions de céréale entière et d'aliment complet ou d'aliment complémentaire ont été mis au point. Aux Pays-Bas et en Belgique, les quantités de blé et d'aliment complémentaire distribuées sont commandées quotidiennement par un système informatique gérant une bascule électronique. L'évolution des proportions est programmée en fonction de l'âge des poulets, l'addition du blé entier dans le régime se faisant à partir de 8 à 15 jours d'âge, avec une incorporation variant entre 10 et 40 à $50 \%$ du régime global, en fonction de l'âge et de la formulation de l'aliment complémentaire (Montjoie 1995, Le Douarin 1997). Au Danemark et en Grande-Bretagne, le système «Flockman " est fondé plus particulièrement sur les besoins quotidiens en lysine, en fonction du poids réel des poulets, évalué à partir de perches-pesons placés dans le poulailler et reliés au système informatique, et de la consommation réelle journalière. L'incorporation maximale de blé est de $15 \%$ en moyenne avec une gamme d'aliments complets (Filmer 1991, 1995 et 1996, Le Boucher 1994, Le Douarin 1997).

La technique de mélange permet de contrôler la composition de l'ingéré global, et de la moduler en fonction de l'âge des poulets et des performances de croissance. Cependant, les poulets peuvent opérer à un tri particulaire. De plus, les équipements automatisés de mélange sont coûteux (Le Douarin 1997) et les contrôles automatiques des poids des poulets utilisés dans le cadre de la régulation automatique des quantités d'aliments distri- 
buées sont peu fiables pendant la phase de finition et ne semblent donc pas adaptés pour ajuster le mélange.

\section{3 / La technique d'alimentation séquentielle}

Le principal avantage de cette méthode est la simplicité de sa mise en œuvre pratique puisque les mêmes installations de distribution alimentaire qu'avec un régime complet sont utilisées. Elle nécessite comme les autres un silo supplémentaire pour les céréales. Un système d'inversion de distribution des deux aliments programmé par une minuterie s'ajoute par rapport au matériel nécessaire en alimentation séparée. Avec un aliment complet de type "démarrage " offert pendant 18 heures, suivi de blé complet pendant 6 heures, des poulets consomment en moyenne $15 \%$ de blé entier dans leur ration globale entre 1 et 49 jours d'âge (Covasa et Forbes 1994). Avec des séquences d'égales durées pour les deux types d'aliment ( 8 heures), et un aliment formulé à partir d'un aliment complet duquel on a ôté la moitié du

Figure 3. Alimentation séquentielle : effet de la durée des séquences de distribution du blé et de l'aliment complémentaire sur le pourcentage de blé consommé en moyenne hebdomadaire (Rose et al 1995).

Dans cette expérience, les poulets sont élevés sous éclairage permanent. Ils ont accès en alternance, pour la même durée totale, soit la moitié du temps, au blé en graines entières et à l'aliment complémentaire. Les différences entre traitements concernent donc uniquement la durée des phases soit, sur $48 \mathrm{~h}, 12$ phases de $4 \mathrm{~h}$, 6 phases de $8 \mathrm{~h}, 4$ phases de $12 \mathrm{~h}$ ou 2 phases de $24 \mathrm{~h}$. Le pourcentage de blé consommé diffère de $50 \%$ et augmente avec l'âge. Pour des cycles de $4 \mathrm{~h}$ le poulet tend à sous-consommer le blé. II n'y a donc pas proportionnalité entre durée et consommation. D'autre part, des périodes courtes de distribution du blé graines entières semblent limiter la consommation.

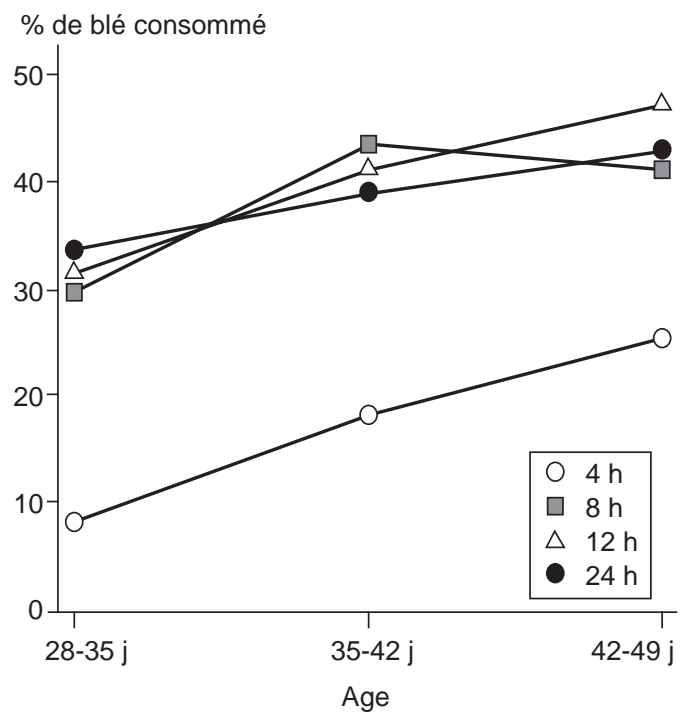

blé (30\% de protéines, $3060 \mathrm{kcal} / \mathrm{kg}$ ), les poulets consomment, entre 28 et 49 jours d'âge, $44 \%$ de leur ration sous forme de céréales entières (Rose et al 1995). Ces deux variantes de l'alimentation séquentielle permettent d'obtenir des performances de croissance comparables à celles obtenues avec un aliment complet.

Si les séquences sont trop courtes (4 heures, par exemple), le poulet peut exprimer un rejet de la céréale et jeûner jusqu'à la séquence " aliment complémentaire » qui suit. Des séquences supérieures à $12 \mathrm{~h}$ peuvent mettre l'animal en situation de carence nutritionnelle (figure 3). Il semble qu'une durée intermédiaire, de l'ordre de 8 heures, soit préférable (Rose et al 1995). Un approfondissement des recherches est nécessaire pour définir les durées optimales des séquences, selon l'âge de l'animal. Le principal inconvénient de cette technique est que l'on manque encore d'expérience de terrain pour valider les paramètres de temps des distributions dans les conditions réelles de l'élevage : il faut tenir compte de l'inertie des chaînes de distribution et des capacités d'ajustement des poulets, qui ne consomment pas l'aliment proposé strictement proportionnellement au temps. Le programme lumineux doit aussi être pris en considération.

Les avantages et inconvénients des trois méthodes sont résumés au tableau 1 . La distribution séquentielle, comme le mélange, semble permettre d'obtenir les performances de croissance les plus proches de celles mesurées avec un aliment complet. Ces techniques seraient donc les mieux adaptées aux élevages industriels européens pour valoriser les céréales entières. Dans l'état actuel de sa mise en œuvre, la technique de l'alimentation séparée, quant à elle, ne permet pas toujours au poulet, de se constituer spontanément un régime optimum au sens des intérêts de l'éleveur.

\section{2 / Résultats récents et perspectives de recherche}

Les recherches menées actuellement sur l'utilisation des céréales entières vont au-delà de la simple mesure des performances de croissance dans différentes conditions expérimentales. Elles analysent aussi les effets de l'utilisation des graines entières sur la nutrition, le comportement et la santé animale.

\section{1 / Nutrition}

\section{a / Forme de présentation des deux types d'aliments}

En libre choix, la forme de présentation des deux aliments proposés intervient en plus de leurs caractéristiques nutritionnelles dans la sélection alimentaire chez le poulet. Elle modifie les proportions de céréales (blé ou

\section{L'alimentation séquentielle donne des résultats comparables à ceux obtenus avec un aliment complet. La durée des séquences de distribution doit être adaptée à l'âge des poulets.}


Tableau 1. Avantages et inconvénients des trois principales méthodes de distribution de céréales graines entières. L'analyse des principaux résultats des recherches portant sur les techniques de distribution de céréales entières sur les 15 dernières années permet de dégager les avantages et les inconvénients de chacune en termes de principes généraux, performances de croissance obtenues et mise en œuvre pratique en élevage.

\begin{tabular}{|c|c|c|c|}
\hline & $\begin{array}{l}\text { Alimentation } \\
\text { séparée }\end{array}$ & Mélange & $\begin{array}{l}\text { Alimentation } \\
\text { séquentielle }\end{array}$ \\
\hline $\begin{array}{l}\text { Avantages } \\
\text { Principe }\end{array}$ & $\begin{array}{l}\text { On obtient des rations } \\
\text { individualisées théoriquement } \\
\text { adaptées aux conditions } \\
\text { d'ambiance et aux potentiels } \\
\text { individuels de croissance } \\
\text { (Mastika et Cumming 1987). }\end{array}$ & $\begin{array}{l}\text { Le choix est dirigé grâce } \\
\text { au contrôle des proportions } \\
\text { des deux aliments, } \\
\text { avec la possibilité de les faire } \\
\text { évoluer en fonction de l'âge } \\
\text { du poulet. }\end{array}$ & $\begin{array}{l}\text { Le choix est dirigé grâce } \\
\text { à la modulation de la durée } \\
\text { des séquences d'accès } \\
\text { à chaque type d'aliment. }\end{array}$ \\
\hline Résultats & $\begin{array}{l}\text { Les performances sont } \\
\text { au mieux égales } \\
\text { à celles obtenues avec } \\
\text { un régime complet équilibré. }\end{array}$ & $\begin{array}{l}\text { Les performances } \\
\text { de croissance sont comparables } \\
\text { à celles obtenues } \\
\text { avec un aliment complet. }\end{array}$ & $\begin{array}{l}\text { Les performances } \\
\text { de croissance sont comparables } \\
\text { à celles obtenues } \\
\text { avec un aliment complet. }\end{array}$ \\
\hline $\begin{array}{l}\text { Mise en œuvre } \\
\text { pratique }\end{array}$ & $\begin{array}{l}\text { Elle est simple } \square \text { : seules } \\
\text { deux chaînes séparées } \\
\text { d'alimentation } \\
\text { sont nécessaires. }\end{array}$ & $\begin{array}{l}\text { Un ajustement rapide } \\
\text { des proportions de céréale } \\
\text { et d'aliment complémentaire } \\
\text { est possible en fonction } \\
\text { des performances. }\end{array}$ & $\begin{array}{l}\text { La réalisation pratique est } \\
\text { simple avec un système } \\
\text { d'horloge et deux silos de } \\
\text { stockage. }\end{array}$ \\
\hline \multicolumn{4}{|l|}{ Inconvénients } \\
\hline Principe & $\begin{array}{l}\text { Les nombreux facteurs } \\
\text { intervenant dans la sélection } \\
\text { alimentaire sont difficiles } \\
\text { à analyser et à maitriser. }\end{array}$ & $\begin{array}{l}\text { Les poulets peuvent opérer } \\
\text { un tri particulaire variable } \\
\text { d'un individu à l'autre. }\end{array}$ & $\begin{array}{l}\text { Il reste à déterminer la durée } \\
\text { optimale de chaque type de } \\
\text { séquence pour un âge donné. }\end{array}$ \\
\hline Résultats & $\begin{array}{l}\text { Les résultats sont très } \\
\text { variables en termes de } \square \text { : } \\
\text { - proportions des deux types } \\
\text { d'aliment sélectionnés } \\
\text { - ratio énergie/protéines } \\
\text { - performances de croissance } \\
\text { - qualité des carcasses } \\
\text { (augmentation de la teneur } \\
\text { en gras et/ou diminution } \\
\text { du rendement en filets). }\end{array}$ & $\begin{array}{l}\text { Les contrôles automatiques } \\
\text { des poids des poulets } \\
\text { fonctionnent mal en finition } \\
\text { et ne peuvent donc pas être } \\
\text { fiables et utilisés pour ajuster } \\
\text { le mélange. }\end{array}$ & $\begin{array}{l}\text { La consommation n'est pas } \\
\text { strictement proportionnelle au } \\
\text { temps d'accès. }\end{array}$ \\
\hline $\begin{array}{l}\text { Mise en œuvre } \\
\text { pratique }\end{array}$ & $\begin{array}{l}\text { Il reste à déterminer l'espace } \\
\text { relatif à occuper par chacun } \\
\text { des deux aliments } \\
\text { dans les conditions } \\
\text { de l'élevage et la distance } \\
\text { entre les chaînes. }\end{array}$ & $\begin{array}{l}\text { Les équipements de mélange } \\
\text { et l'automatisation } \\
\text { informatique sont coûteux } \\
\text { (Filmer 1991, } \\
\text { Le Douarin 1997). }\end{array}$ & $\begin{array}{l}\text { La technique doit être ajustée } \\
\text { au programme lumineux } \\
\text { de chaque élevage. }\end{array}$ \\
\hline
\end{tabular}

maïs) et d'aliment complémentaire consommées (Rose et al 1986, Yo et al 1998). Des textures proches (blé et complémentaire en farine, ou blé entier et complémentaire granulé de diamètre $5 \mathrm{~mm}$ ) semblent favoriser une consommation spontanée de céréales élevée (figure 4). Il serait nécessaire d'analyser l'effet des formes physiques en distribution séquentielle.

\section{b / Age à l'introduction de la céréale et apprentissage}

L'apprentissage est un des facteurs influençant la sélection alimentaire. Jusqu'à 3 à 5 jours d'âge, l'association des caractéris- tiques sensorielles de l'aliment et de ses conséquences nutritionnelles se fait de façon imprécise car le développement du cerveau n'est pas achevé, et la persistance du résidu vitellin interfère avec la perception des besoins nutritionnels exogènes (Covasa et Forbes 1996). Après une semaine d'âge, l'âge absolu d'introduction de la céréale en alimentation séparée ne semble pas modifier la durée de la période d'adaptation nécessaire pour constituer un régime globalement équilibré (Yo et al 1997). Cette période est variable : elle est fonction des différences de présentation et de composition du régime précédant le choix entre une céréale et un aliment complémentaire (Rose et Kyriasakis 1991). 


\section{c / Teneur en énergie et forme de présentation}

La forme de présentation de la céréale influence peu l'efficacité de sa digestion mesurée par test de bilan. La teneur en énergie métabolisable à bilan azoté nul (EMAn) du blé mesurée sur des jeunes poulets de chair est la même, que le blé soit présenté sous forme de grain entier, de farine ou de granulés. En revanche, pour le maïs, elle est inférieure de $120 \mathrm{kcal} / \mathrm{kg}$ de matière sèche pour le maïs en grain entier par rapport à la farine ou aux granulés (Barrier-Guillot et al 1997). Dans le cas du blé, la digestibilité de l'énergie ne semble pas influencée par la dureté de l'endosperme, lorsque l'on compare deux variétés

Figure 4. Alimentation séparée : effet de la présentation de l'aliment sur le niveau de consommation hebdomadaire de blé entier par des poulets (d'après Rose et al 1986).

Des poulets en alimentation séparée entre 21 et 49 jours d'âge, recevant du blé entier ou en farine avec un complémentaire en granulés ou en farine, consomment dans un premier temps plus de blé s'il est sous forme de farine. En quelques jours, ils s'adaptent à l'ingestion et à la digestion des aliments particulaires. Dans le cas « blé farine + complémentaire granulé ", l'ingestion de l'aliment protéique augmente avec le temps et l'ingestion d'énergie (blé) diminue. A l'inverse, les poulets recevant des régimes avec du blé entier ont une consommation croissante de blé avec l'âge, plus marquée si le complémentaire est granulé. La proportion de blé sélectionnée dans le régime est significativement influencée $(P<0,01)$ par l'interaction entre les deux formes de présentation des aliments. L'efficacité alimentaire moyenne n'est pas affectée par les formes respectives de présentation des aliments, mais une consommation importante de blé entraîne une augmentation de la teneur en gras abdominal des carcasses dans cette expérience.

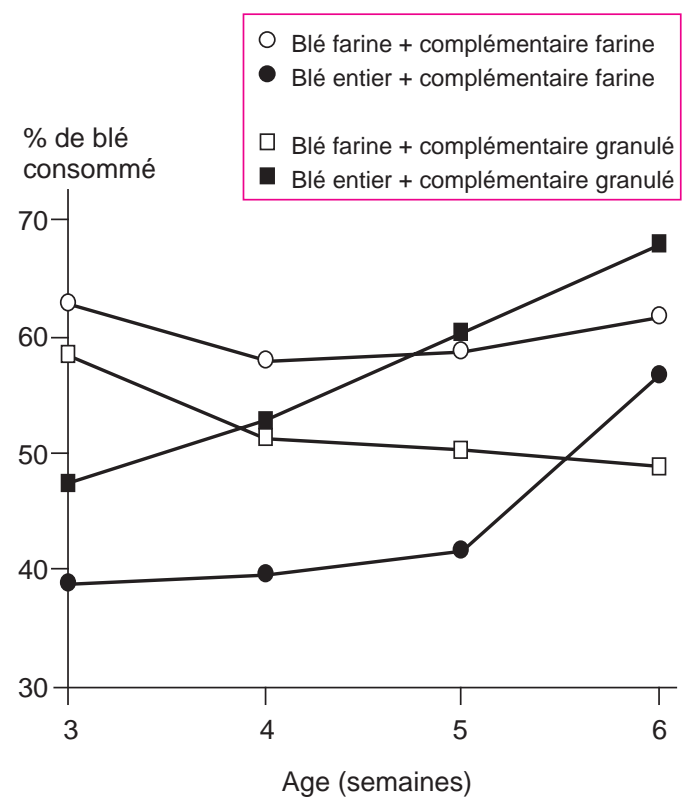

de dureté différente, que le blé soit offert entier avec un aliment complémentaire ou incorporé dans un aliment complet (Rose 1996).

\section{2 / Adaptation comportementale}

Les poulets en alimentation séparée expriment un comportement alimentaire différent de ceux recevant un aliment complet. En alimentation séparée, les accès aux deux types de mangeoire (maïs et aliment complémentaire) sont plus courts et plus nombreux que ceux consacrés à l'aliment complet. L'accès aux deux aliments n'est pas aléatoire : à 30 jours, les accès aux mangeoires de maïs entier sont deux fois plus nombreux que ceux aux mangeoires d'aliment complémentaire. Le poulet module donc la fréquence d'accès aux deux aliments pour réguler son ingestion énergétique et protéique, et il est capable d'identifier l'aliment par la mémorisation de sa localisation dans le lieu de vie (Yo 1996).

A court terme, le choix se fait en premier lieu sur les caractéristiques sensorielles de l'aliment, puis il est modifié en fonction de l'apprentissage des conséquences post-ingestives de l'absorption des deux types d'aliment. Des poulets « naïfs " picorent à un rythme plus lent que leurs congénères expérimentés, ce qui suggère une plus longue période d'observation de l'aliment entre deux coups de becs consécutifs (Yo et al 1998).

En alimentation séquentielle, l'introduction du blé ou de l'aliment complémentaire stimule l'activité alimentaire des poulets pendant la période qui suit l'introduction du nouvel aliment, alors que les poulets recevant un aliment unique ou soumis à une alimentation séparée dans l'espace montrent peu de variations diurnes de leur activité alimentaire (Foote et Rose 1991).

\section{3 / Santé}

\section{a / Conséquences du développement du gésier}

L'alimentation actuelle du poulet de chair, riche en énergie et pauvre en fibres, en l'absence de distribution de grit, ne stimule pas le développement du gésier, et les particules alimentaires arrivent peu broyées dans le duodénum. Un régime comportant des céréales entières rend au gésier son rôle d'organe de broyage, et il semblerait qu'un gésier fonctionnel permette de détruire en partie mécaniquement les oocystes. L'excrétion d'oocystes chez le mâle est négativement corrélée à la taille du gésier, quel que soit le type d'alimentation (Cumming 1992). Le poids du gésier est augmenté en moyenne de $1 \%$ du poids de la carcasse par la consommation de graines entières de céréales (Scholtyssek et al 1983, Munt et al 1995).

L'action des enzymes digestives et du $\mathrm{pH}$ bas du proventricule, suivie d'une rétention des particules de céréales pendant une

\section{Les formes de présentation de la céréale et de l'aliment complémentaire ont un effet sur la quantité de céréale consommée.}


période plus longue dans un gésier mieux développé permettrait aux aliments d'arriver partiellement digérés dans le duodénum, ce qui réduirait la prolifération de bactéries pathogènes telles que $E$. coli dans l'intestin (Cumming 1992). Les conséquences physiologiques du développement du gésier chez le poulet de chair mériteraient des études plus précises.

\section{b / Dilution des additifs alimentaires dans le régime céréales + complémentaire}

Les additifs alimentaires contenus dans l'aliment complémentaire, sont dilués dans le régime global par l'apport de céréales entières. Or les quantités maximales de coccidiostatiques et d'antibiotiques que l'on peut ajouter aux aliments sont réglementées (directive européenne 90/643). Pour la plupart des antibiotiques, l'intervalle entre les niveaux minimum efficace et maximum réglementaire est suffisamment large pour que les quantités requises soient incorporées dans l'aliment complémentaire. En revanche, pour certains coccidiostatiques comme l'Arpinocide et le Méticlorpindol, la marge est nulle. Le niveau d'incorporation se trouve donc toujours inférieur au minimum requis. Le recours aux traitements dans l'eau de boisson peut être nécessaire, car la résistance accrue aux coccidies et autres troubles digestifs avec un gésier fonctionnel reste hypothétique.

Les observations en élevage ne révèlent pas de problèmes particuliers de coccidioses avec du blé entier. Dans les pays scandinaves ou dans d'autres pays européens où le blé entier est incorporé à de l'aliment complet titrant $24 \%$ de protéines brutes, à partir de 8 jours d'âge, et en proportion croissante jusqu'à $40 \%$ à partir de 21 jours, les éleveurs ne semblent pas constater d'augmentation de l'incidence des coccidioses, malgré la dilution des anticoccidiens dans le régime global (Cumming 1992).

Les travaux de recherche récents montrent que le poulet est capable de s'adapter à un régime céréale entière + aliment complémen- taire. Il modifie son comportement alimentaire, sans période d'apprentissage, à condition que l'aliment proposé avant l'introduction de la céréale entière facilite la transition alimentaire. La digestion est améliorée par rapport à un aliment complet.

\section{Conclusion}

L'incorporation de céréales entières dans l'alimentation du poulet de chair entraîne la distribution de deux types d'aliments, dont les proportions ne peuvent être que partiellement contrôlées, car les différents facteurs individuels de sélection alimentaire interviennent. La recherche s'est principalement concentrée sur l'étude de l'alimentation séparée dans l'espace, mais, si le poulet est capable de constituer un régime globalement équilibré, celui-ci ne correspond pas toujours à la croissance et à la composition corporelle optimales recherchées par l'éleveur. Les techniques du mélange et de l'alimentation séquentielle permettent de mieux réguler les taux d'ingestion des deux types d'aliment, mais n'excluent pas la manifestation d'une préférence de l'animal pour l'un ou l'autre. La distribution en mélange donne de bons résultats dans les élevages d'Europe du nord, mais nécessite un investissement relativement coûteux. L'alimentation séquentielle demande moins d'équipements et présente l'attrait de la simplicité. Elle est encore incomplètement connue sur le plan scientifique et nécessite plusieurs ajustements pratiques.

Le succès des techniques de distribution des céréales dépend de la formulation de l'aliment complémentaire en protéines et en additifs, selon le niveau d'ingestion des céréales entières. En alimentation séquentielle, des recherches restent à mener pour déterminer la durée et le moment de distribution des deux aliments en fonction de l'âge et du programme lumineux. Une fois les techniques de distribution maîtrisées et validées, l'avenir de l'utilisation des céréales entières dans l'alimentation du poulet de chair repose sur son intérêt économique réel pour l'éleveur, sachant que l'image du " poulet nourri au grain » peut être valorisée commercialement.

\section{Références}

Barrier-Guillot B., Métayer J.P., Bouvarel I., Castaing J., Picard M., Zwick J.L., 1997. Valeur énergétique du blé et du maïs présentés en grains entiers, en farine et en granulé chez le poulet de chair. $2^{\text {es }}$ journées de la Recherche Avicole, ITAVI, Paris (FRA), 37-39.

Covasa M., Forbes J.M., 1994. Performances of broiler chickens as affected by split time feeding and wheat diluted diet. Proc. 9th European Poultry Conf., WPSA United Kingdom Branch, Roslin (UK), vol 1, 457-458.

Covasa M., Forbes J.M., 1995. Application of diet selection by poultry with particular reference to whole cereals. World Poult. Sci. J., 51, 149-165.
Covasa M., Forbes J.M., 1996. Effects of prior experience and training on diet selection of broiler chickens using wheat. Appl. Anim. Behav. Sci., 46, 229242 .

Cowan P.J., Mitchie W., 1978. Choice feeding of the male and female broiler. Br. Poult. Sci., 19, 149-152.

Cumming R.B., 1992. The advantages of free choice feeding for village chicken. Proc. of the 19th World Poultry Congress, WPSA Dutch Branch, Odijk (NL), vol 3, 627-632.

Emmans G.C., 1991. Diet selection by animals : theory and experimental design. Proc. Nutr. Soc., 50, $59-64$. 
Filmer D., 1991. A new system for livestock feeding. Feeds \& Feeding, July/August 1991, 30-33.

Filmer D., 1995. Market share. Flockman Newsletter $\mathrm{n}^{\circ} 1$, Dec 1995, 1-2.

Filmer D., 1996. Wheat or low protein ? Flockman Newsletter $n^{\circ} 2$, Nov 1996, 1-2.

Foote W.R., Rose S.P., 1991. Sequential feeding of whole grain wheat to broilers. Br. Poult. Sci., 32, 1131-1132.

Forbes J.M., Kyriasakis I., 1995. Food preferences in farm animals : why don't they always choose wisely? Proc. Nut. Soc., 54, 429-440.

Gérard C., 1997. En Belgique, le poulet valorise le blé. Réussir Aviculture, 26, 20-22.

Le Boucher G., 1994. Du blé entier dans l'aliment des poulets. Filières Avicoles, déc 1994, 42-44.

Le Douarin P., 1997. Système Flockman : le blé entier ajuste la ration. Réussir Aviculture, 26, 23-24.

Leeson S., Caston L.J., 1993. Production and carcass yield of broilers using free-choice cereal feeding. J. Appl. Poult. Res., 2, 253-258.

Mastika M., Cumming R.B., 1987. Effect of previous experience and environmental variations on the performance and pattern of feed intake of choice fed and complete fed broilers. In : D.J. Farrel (ed), Recent advances in animal nutrition in Australia, 260-282. Univ. of New England (AUS).

Meunier-Salaün M.C., Picard M., 1996. Les facteurs de choix alimentaires chez le porc et les volailles. INRA Prod. Anim., 339-348.

Montjoie Y., 1995. Une technique d'alimentation courante aux Pays-Bas : du blé entier pour abaisser le prix de revient du poulet. Filières Avicoles, déc 1995, 64-67.

Munt R.H.C., Dingle J.G., Sumpa M.G., 1995. Growth, carcass composition and profitability of meat chickens given pellets, mash or free choice diet. Br. Poult. Sci., 36, 277-284.

Picard M., 1997. Broiler behaviour and nutritional conditions. 11th European Symposium on Poultry Nutrition, WPSA Danish Branch, Copenhagen (DK), 175-180.
Rose S.P., 1996. The use of whole wheat in poultry diets. World's Poult. Sci. J., 52, 59-60.

Rose S.P., Kyriasakis I., 1991. Diet selection of pigs and poultry. Proc. Nut. Soc., 50, 87-98.

Rose S.P., Lambie I.T.M., 1986. Comparison of a choice feeding regime for broilers under continuous and intermittent lighting programmes. $7^{\mathrm{e}}$ Conférence Européenne d'Aviculture, WPSA Branche française, St Cyr sur Loire (FRA), vol 2, 903-904.

Rose S.P., Burnett A., Elmajeed R.A., 1986. Factors affecting the diet selection of choice feed broilers. Br. Poult. Sci., 27, 215-224.

Rose S.P., Fielden M., Foote W.R., Gardin P., 1995. Sequential feeding of whole wheat to growing broiler chickens. Br. Poult. Sci., 36, 97-111.

Scholtyssek S., Seemann M., Seemann G., 1983. Mastleistung und Schlachtkörperqualität nach Wahlfütterung von Broilern (I. Mitteilung). Arch. Geflügelk., 47, 166-174.

Siegel P.B., Picard M., Nir I., Dunnington E.A., Willemsen M.H.A., Williams P.E.V., 1997. Responses of meat-type chickens to choice feeding of diets differing in protein and energy from hatch to market weight. Poult. Sci., 76, 1183-1192.

Yo T., 1996. Adaptation comportementale au choix alimentaire du poulet de chair et de la poule pondeuse (Gallus domesticus) en milieu tropical. Thèse Ecole Nationale Supérieure Agronomique de Rennes (FRA), $265 \mathrm{p}$.

Yo T., Picard M., Guérin H., Dauvilliers P., 1994. Alimentation séparée (céréales entières + aliment complémentaire granulé) chez les poulets de chair en climat chaud. Revue Elev. Méd. vét. Pays trop., 47, 319-327.

Yo T., Siegel P.B., Guérin H., Picard M., 1997. Self selection of dietary protein and energy by broilers grown under a tropical climate : effect of feed particle size on the feed choice. Poult. Sci., 76, 14671473.

Yo T., Siegel P., Faure J.M., Picard M., 1998. Self selection of dietary protein and energy by broilers grown under a tropical climate : adaptation when exposed to choice feeding at different ages. Poult. Sci., 77, 502-508.

\section{Abstract}

Whole cereal grains in broiler nutrition : the come back?

Successes encountered in some northern European poultry farms raise interest for the use of whole cereals grains distributed with a protein concentrate in broiler chicken feeding. The three main types of distribution of whole grains are compared : splitted diets, loose-mix and sequential feeding. Loose-mix and sequential feeding techniques are efficient. These feeding systems allow to control partly the whole cereal and protein concentrate proportions actually ingested by chickens. On the other hand, splitted diets favour the expression of individual factors of food selection, and dœs not always allow to get growth and body composition as expected by poultry farmers.

Broilers digest whole grain as efficiently as ground cereals. Broilers are able to quickly adapt to a diet including a whole cereal grain, by adjust- ment of their feeding behaviour. The proportion of cereal grain ingested depends on the physical form and composition of the protein concentrate. Whole cereal grains induce a larger development of gizzard whose effects on digestion and on natural resistance of the birds towards coccidiosis require further research.

The system of loose-mix feeding is mastered and applied in some northern European poultry farms by computer monitored distribution. Sequential feeding is less expensive than loose-mix feeding but remains to be validated in practise. Economical interest of this feeding method for French farmers requires further evaluation.

Noirot V., Bouvarel I., Barrier-Guillot B., Castaing J., Zwick J.L., Picard M., 1998. Céréales entières pour les poulets de chair : le retour? INRA Prod. Anim., 11, 349-357. 